\title{
A wide-field spectroscopic survey of the cluster of galaxies $\mathrm{Cl} 0024+1654$
}

\section{A high-speed collision?}

\author{
O. Czoske ${ }^{1,2}$, B. Moore ${ }^{3}$, J.-P. Kneib ${ }^{1}$, and G. Soucail ${ }^{1}$ \\ 1 Observatoire Midi-Pyrénées, UMR5572, 14 Av. Édouard Belin, 31400 Toulouse, France \\ 2 Institute for Astronomy, University of Hawaii, 2680 Woodlawn Drive, Honolulu HI 96822, USA \\ 3 Department of Physics, Durham University, South Road, Durham, DH1 3LE, UK
}

Received 9 November 2001 / Accepted 8 February 2002

\begin{abstract}
The mass distribution of the rich cluster of galaxies Cl0024+1654 has frequently been used to constrain the nature of dark matter, yet a model consistent with all the observational data has been difficult to construct. In this paper we analyse the three-dimensional structure of this cluster using new spectroscopic information on $\sim 300$ galaxies within a projected radius of $3 h^{-1} \mathrm{Mpc}$. These data reveal an unusual foreground component of galaxies separated from the main cluster by $3000 \mathrm{~km} \mathrm{~s}^{-1}$. We use numerical simulations to show that a high speed collision along the line of sight between Cl0024+1654 and a second cluster of slightly smaller mass can reproduce the observed peculiar redshift distribution. Such a collision would dramatically alter the internal mass distribution of the bound remnants, creating constant density cores from initially cuspy dark matter profiles and scattering galaxies to large projected radii, consistent with the observed distribution of galaxies in Cl0024+1654. The proposed scenario can reconcile the inferred mass profile from gravitational lensing with predictions from hierarchical structure formation models, while at the same time resolving the mass discrepancy that results from a comparison between lensing, velocity dispersion and X-ray studies.
\end{abstract}

Key words. galaxies: clusters: individual: C10024+1654 - cosmology: observations - cosmology: large-scale structure of Universe

\section{Introduction}

The nature of the ubiquitous dark matter remains one of the major mysteries in cosmology. The traditional view is that the dark matter is cold, i.e. non-relativistic at the decoupling epoch, and only subject to gravitational and weak interactions. Structure formation with this type of dark matter has been investigated extensively through numerical simulations over the past 25 years. High-resolution simulations of individual cold dark matter halos have shown that the mass distribution in CDM halos over a wide range of total masses, from dwarf galaxy to cluster scales, should follow a universal profile (Navarro et al. 1997, NFW); in particular, the mass distribution is found to have a central cusp with logarithmic slope of -1.5 (Moore et al. 1998; Ghigna et al 2000). Recent observational results, however, have challenged this simple picture.

Send offprint requests to: O. Czoske, e-mail: oczoske@ast.obs-mip.fr
The rich cluster of galaxies $\mathrm{Cl} 0024+1654$ at $z=$ 0.395 features a spectacular quintuple gravitational arc system of radius $106 h^{-1} \mathrm{kpc}$ that has been used to reconstruct the projected central mass distribution (Kassiola et al. 1992; Smail et al. 1996; Tyson et al. 1998; Broadhurst et al. 2000). Constructing a mass model for this cluster that is consistent with all the observations has proved very difficult, however. The total projected mass enclosed within the arc radius is fairly well constrained and yields a value of about $1.6 \times 10^{14} h^{-1} M_{\odot}$. To reproduce this mass with an NFW type profile would require a cluster with characteristic velocity dispersion larger than $2000 \mathrm{~km} \mathrm{~s}^{-1}$ (Broadhurst et al. 2000; Shapiro \& Iliev 2000). This is much higher than observed in this or indeed in any other cluster of galaxies. The mass reconstruction by Tyson et al. (1998), based on the detailed structure of the gravitationally lensed background spiral galaxy, shows a flat core in the projected central mass distribution with a core radius of $35 h^{-1} \mathrm{kpc}$. This apparent contradiction to the predictions of standard CDM simulations has prompted a 
number of authors to investigate alternatives to the classic CDM scenario, e.g. warm dark matter, self-interacting dark matter, etc. (Spergel \& Steinhardt 2000; Hogan \& Dalcanton 2000; Moore et al. 2000).

As more detailed observations of individual clusters of galaxies are compiled, combinations of X-ray imaging and spectroscopy, velocity dispersion measurements and lensing mass maps frequently reveal that clusters that were thought to be simple relaxed objects are actually more complex systems, frequently undergoing mergers or generally showing signs of substructure and deviations from dynamical equilibrium. Evidence for recent mergers or accretion is currently accumulating through CHANDRA observations in the form of merger shocks (e.g. Markevitch \& Vikhlinin 2001; Markevitch et al. 2001) or cold fronts (Markevitch et al. 2000; Vikhlinin et al. 2001; Mazzotta et al. 2001). Mergers and substructure have also been invoked to explain the discrepancy between mass estimates from different methods that is observed in many clusters (Miralda-Escudé \& Babul 1995; Wu et al. 1998).

$\mathrm{Cl} 10024+1654$ is an example of a cluster where the high mass inferred from the strong lensing mass reconstructions is at variance with the fairly low X-ray luminosity and temperature, that indicate a total mass for the cluster which is a factor 2 to 3 smaller than the lensing mass (Soucail et al. 2000). Both the galaxy distribution on the sky and the X-ray morphology are regular and by themselves compatible with a relaxed massive cluster, a notion which was further supported by the high galaxy velocity dispersion of $\approx 1200 \mathrm{~km} \mathrm{~s}^{-1}$ found in the redshift surveys of Dressler \& Gunn (1992) and Dressler et al. (1999, hereafter D99).

In Czoske et al. (2001, Paper I) we presented a new catalogue of 650 measured redshifts (including those from D99) in a wide field around Cl0024+1654. 300 galaxies in the catalogue have redshifts in the range $0.37<z<0.42$, i.e. are cluster members or lie in the immediate neighbourhood of the cluster, and the redshift distribution of these galaxies was found in Paper I to be distinctly bimodal. In the present paper we analyse the distribution of the galaxies in redshift/real space and present a merger scenario which is able to reconcile all the observations. Section 2 recalls the data used and describes the distribution of the redshifts globally and as a function of position within the survey field, as well as the distribution of spectral types based on equivalent widths of important lines. In Sect. 3, we develop the merger scenario and use numerical simulations to show that it is possible to explain the peculiar three-dimensional distribution of the galaxies in Cl0024+1654. In Sect. 4 we show how the observed structure is able to explain and resolve the discrepancy of mass estimates of Cl0024+1654 derived from different observations. Finally, in Sect. 5 we summarize and conclude.

Throughout this paper we use a Hubble constant $H_{0}=$ $100 h^{-1} \mathrm{~km} \mathrm{~s}^{-1} \mathrm{Mpc}^{-1}$ and assume an Einstein-de Sitter Universe with $\Omega_{\mathrm{M}}=1$ and $\Omega_{\Lambda}=0$. At the redshift of

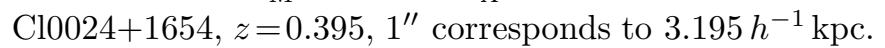

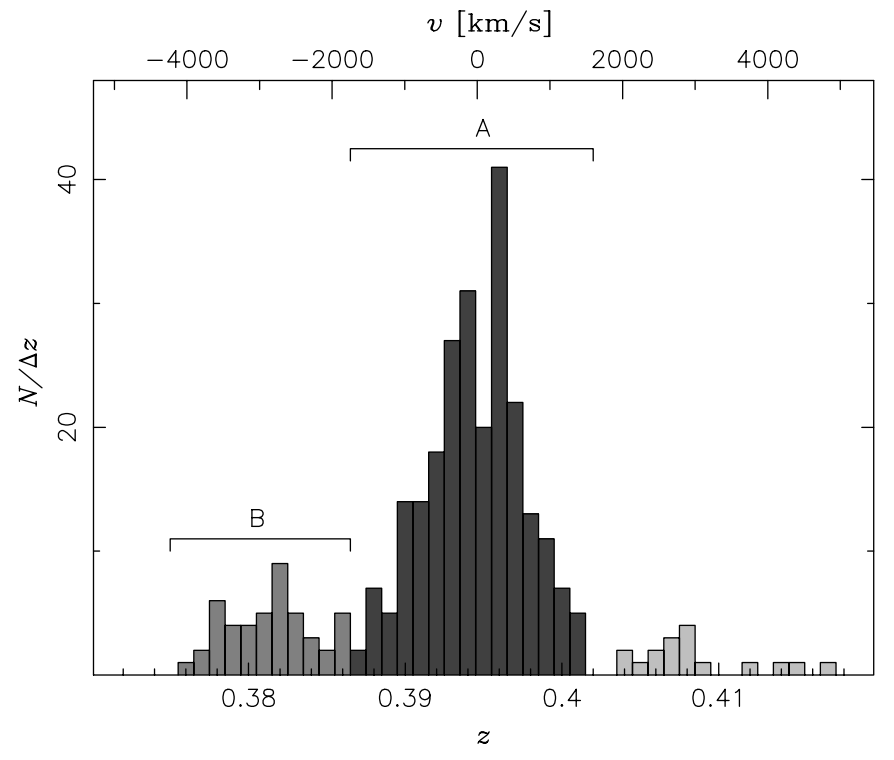

Fig. 1. Redshift histogram for 300 objects in the neighbourhood of $\mathrm{Cl} 0024+1654(0.37<z<0.42)$. The attribution of galaxies to components A (dark grey) and B (light grey) was done by inspection of Fig. 2. Whereas the lower and upper redshift limits of components B and A respectively are fairly secure, the exact demarcation between the two components is arbitrary to some extent and was chosen at $\Delta v=-1500 \mathrm{~km} \mathrm{~s}^{-1}$ with respect to the central redshift of component A, Eq. (2).

\section{The cluster environment}

\subsection{The data}

In Paper I we presented a new catalogue of photometric and spectroscopic data for 679 objects in a wide field of

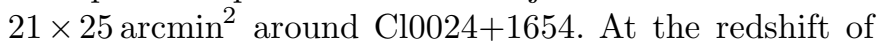
the cluster, $z=0.395$, this corresponds to $4 \times 4.8 h^{-2} \mathrm{Mpc}^{2}$ (the true survey field is delimited by an irregular polygon inscribed in this rectangle and covers $16.8 h^{-2} \mathrm{Mpc}^{2}$, see Fig. 4, or Fig. 7 in Paper I). The catalogue lists equatorial position, redshift, $V$ magnitude, $V-I$ colour, equivalent widths for $[\mathrm{O}$ II $] \lambda 3727,[\mathrm{O}$ III $] \lambda 5007, \mathrm{H} \alpha$ (where within the wavelength range), $\mathrm{H} \beta$ and $\mathrm{H} \delta$, as well as the strength of the $4000 \AA$ break. Here we are only concerned with the 650 objects having measured redshifts, of which 581 were observed by us at CFHT and WHT, with the remaining 69 taken from Dressler et al. (1999). See Paper I for more technical details on the data used in this paper.

\subsection{Redshift distribution}

Figure 1 shows a histogram of the 300 redshifts that lie in the range $0.37<z<0.42$. The redshift distribution of the cluster galaxies is clearly bimodal, showing two peaks at $z=0.381$ and $z=0.395$ respectively; these peaks contain 283 galaxies.

The larger peak at $z=0.395(0.387<z<0.402$, hereafter referred to as component A) contains 237 galaxies, is fairly regular and resembles a Gaussian distribution as expected for a relaxed, virialized cluster of galaxies. 


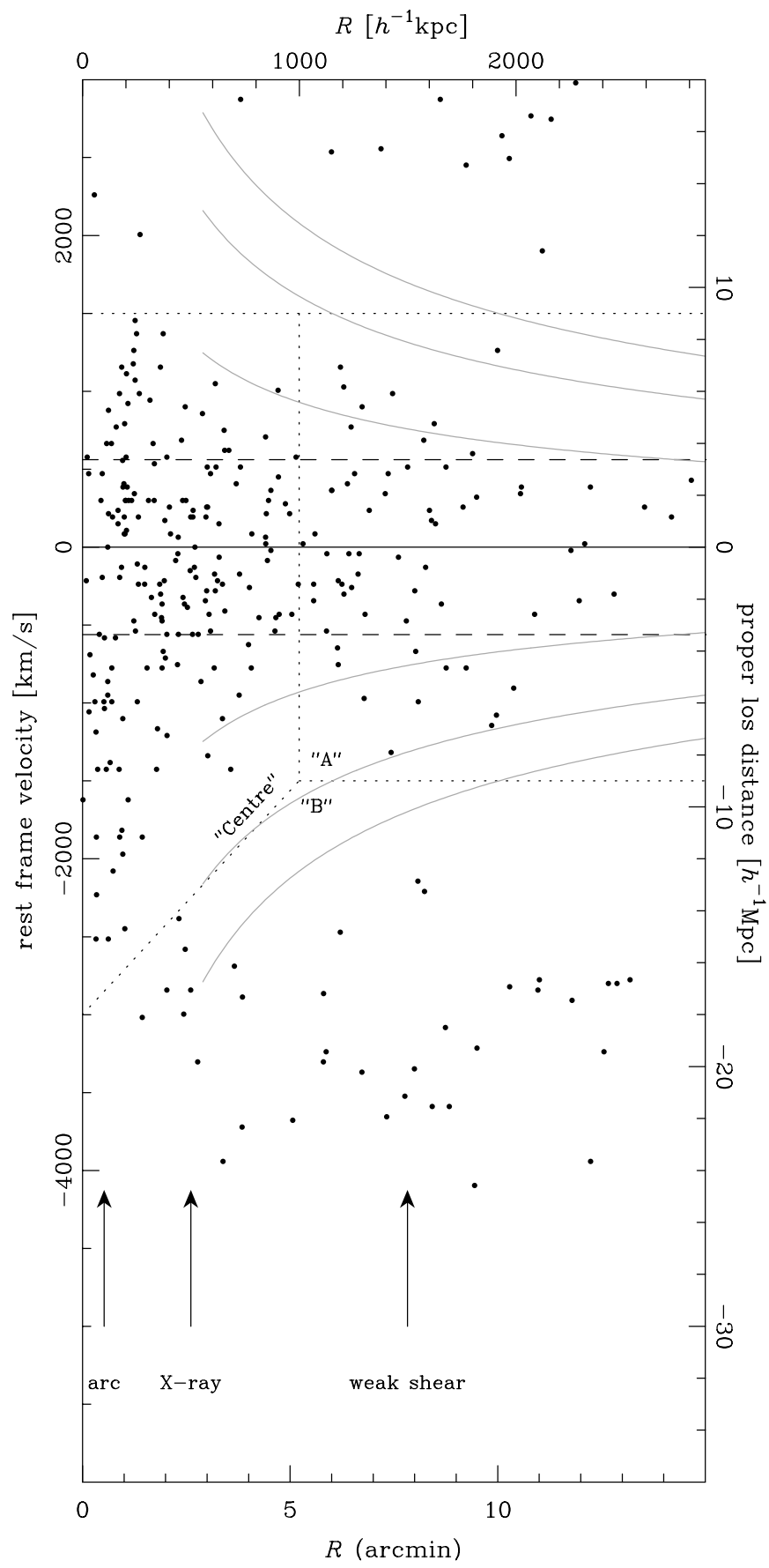

Fig. 2. Redshift $z$ plotted against angular distance $R$ from the projected cluster centre for the galaxies around the cluster redshift. The left axis expresses redshift as relative velocity with respect to the mean redshift of component A (Eq. (2)), the right axis as proper line-of-sight distance at the cluster redshift. Angular distance is converted to proper transverse distance on the top axis. The dashed horizontal lines indicate the velocity dispersion of component A (Eq. (3)), the dotted lines denote limits for the samples used in Sect. 2.2. The solid curves mark the escape velocities for masses inside radius $R$ of 1,3 and $5 \times 10^{14} M_{\odot}$. Arrows mark the position of the giant arc and the X-ray and weak shear detection limits.

The smaller foreground peak at $z=0.381(0.374<z<$ 0.387 , component B), by contrast, seems too wide for the

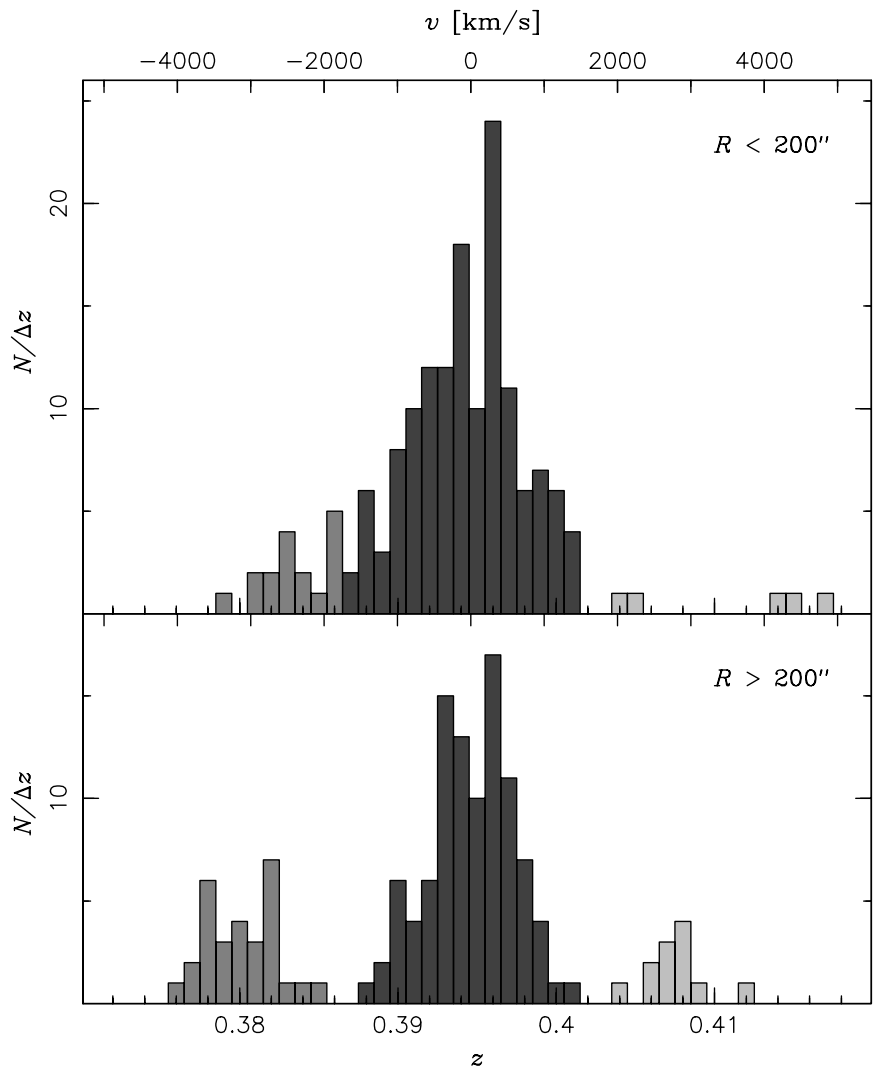

Fig. 3. Redshift histograms for objects inside (upper panel) and outside (bottom panel) a radius of $200^{\prime \prime}$ (corresponding to $640 h^{-1} \mathrm{kpc}$ ) from the projected cluster centre. In the central redshift distribution there is no correspondence to the distinct peak at $z=0.38$ visible in the external distribution.

small number of 46 redshifts contained in it. This impression is confirmed by Fig. 2, which plots the redshift for each galaxy versus its projected distance from the cen-

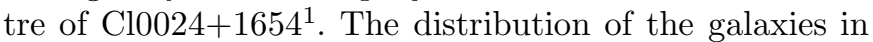
the main peak is symmetrical with respect to the central redshift line, whereas the distribution of the foreground galaxies is roughly constant at a rest frame velocity of $\sim-3000 \mathrm{kms}^{-1}$ at radii larger than $3^{\prime}\left(600 h^{-1} \mathrm{kpc}\right)$, but turns off towards smaller relative velocities to merge with the main distribution at smaller projected distances.

The 17 galaxies in the peak at $z=0.407$ are more widely dispersed across the survey field and although

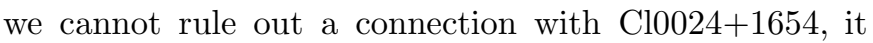
seems more likely, in the light of the scenario developed in Sect. 3, that they are part of the surrounding field galaxy population.

It is remarkable that we can trace the main cluster as well as the foreground structure out to the edge of the survey field (there remains only one object in the catalogue outside the range depicted in Fig. 2,

1 The coordinates in the catalogue are given relative to $\alpha_{2000}=00^{\mathrm{h}} 26^{\mathrm{m}} 35.70, \delta_{2000}=17^{\circ} 09^{\prime} 43^{\prime \prime} \cdot 06$. Originally, this was the position of galaxy 373 , but a more accurate astrometric analysis shifted the reference point by 0.79 to the south-east of this galaxy. 
a background galaxy). The virial radius for Cl0024+1654 should be around $1 h^{-1} \mathrm{Mpc}$ (Girardi \& Mezzetti 2001), which means that we detect a coherent structure out to three times the virial radius.

\section{Redshift distribution inside 200"}

From Figs. 2 and 3 (upper panel) it is obvious that the central region of $\mathrm{Cl} 0024+1654$ is highly perturbed and it is impossible to separate components A and B within 200" $\left(640 h^{-1} \mathrm{Mpc}\right)$ from the projected cluster centre. However, the redshift distribution for the central galaxies is strongly skewed towards negative velocities: except for two galaxies that are fairly isolated in redshift space, the redshift distribution at positive velocities is effectively cut off at $\sim 1500 \mathrm{~km} \mathrm{~s}^{-1}$, whereas the distribution at negative velocities extends to beyond $\sim-2500 \mathrm{~km} \mathrm{~s}^{-1}$, branching off into component B at $\sim 500 h^{-1} \mathrm{kpc}$ from the projected cluster centre. Quantitatively the skewness of the distribution of 161 galaxies within $200^{\prime \prime}(0.37<z<0.42)$ is 0.54 , where we use the definition given by Press et al. (1992):

$\operatorname{Skew}\left(x_{1} \ldots x_{N}\right)=\frac{1}{N} \sum_{j=1}^{N}\left[\frac{x_{j}-\bar{x}}{\sigma}\right]^{3}$.

The probability that a sample of size $N=161$ drawn from a normal distribution shows a skewness larger than that measured in Cl0024+1654 is about $0.3 \%$. The ShapiroWilk normality test (Shapiro \& Wilk 1965) rejects the hypothesis that these data points are drawn from a Gaussian parent population at $\gg 99 \%$ confidence. The skew in the central redshift distribution is already apparent in the histogram given by Dressler et al. (1999) (see also Sect. 4).

\section{Redshift distribution outside $200^{\prime \prime}$}

In the distribution of the galaxies outside 200" (Figs. 2 and 3 , bottom panel) the two modes are clearly separated. The mean redshift of the 71 galaxies belonging to component $\mathrm{A}$ at projected distances $200^{\prime \prime}<R<500^{\prime \prime}$ is

$\overline{z_{\mathrm{A}}}=0.3946 \pm 0.0007$.

In Fig. 5 we show the two velocity dispersion profiles for galaxies in component $\mathrm{A}$ with either positive or negative velocities with respect to the mean redshift $\overline{z_{\mathrm{A}}}$, calculated in a sliding bin containing 30 galaxies. Outside $3^{\prime}$ $\left(600 h^{-1} \mathrm{kpc}\right)$ both profiles are flat at the same level of $\sigma \approx 600 \mathrm{~km} \mathrm{~s}^{-1}$. The profile for galaxies with negative velocities (i.e. those moving towards us, Fig. 5) rises all the way to about $45^{\prime \prime}\left(140 h^{-1} \mathrm{kpc}\right)$, reaching a velocity dispersion of nearly $900 \mathrm{~km} \mathrm{~s}^{-1}$ in the innermost bin. The dispersion profile of the galaxies with positive velocities, by contrast, drops back to a value of about $600 \mathrm{~km} \mathrm{~s}^{-1}$ after having reached a maximum value of about $800 \mathrm{~km} \mathrm{~s}^{-1}$ at $2^{\prime}$ from the centre. Although the galaxy velocities used in Fig. 5 have been restricted to $|v|<1500 \mathrm{~km} \mathrm{~s}^{-1}$, the different behaviour of the profiles for galaxies with positive and negative velocities respectively reflects the skew in the central redshift distribution and indicates the possible presence of a bulk motion component towards us in the central galaxy distribution.

The exact value of the velocity dispersion of the 71 galaxies within the same distance range as used for Eq. (2) and velocity $|v|<1500 \mathrm{~km} \mathrm{~s}^{-1}$ relative to the central redshift $\overline{z_{\mathrm{A}}}$ is

$\sigma_{\mathrm{A}}=561_{-83}^{+95} \mathrm{~km} \mathrm{~s}^{-1}$.

Both $\overline{z_{\mathrm{A}}}$ and $\sigma_{\mathrm{A}}$ were computed using the biweight estimator (Beers et al. 1990), the errors (95\% confidence level) were estimated by bootstrap resampling.

The skewness of the distribution of these 71 redshifts is -0.12 , which should be compared to the standard deviation of skewness for samples of this size drawn from a normal distribution, 0.27 . The Shapiro-Wilk test does not provide evidence for deviations from a Gaussian distribution.

The spatial positions of the galaxies in the foreground component B show no preference for any direction (Fig. 4, middle panel). The formal velocity dispersion for component $\mathrm{B}$ in the same radial distance range as used in Eq. (3) is $\sigma_{\mathrm{B}}=554_{-304}^{+175} \mathrm{~km} \mathrm{~s}^{-1}$ based on 15 redshifts. Given that the number of galaxies in component $\mathrm{A}$ is almost five times as large as the number in component B it is hardly conceivable that both components should have the same velocity dispersion - their mass-to-light ratios would be extremely different. Component B is certainly not a virialized group or cluster.

From the distribution of the external galaxies alone one might still suspect the presence of a loose aggregate of galaxies physically disconnected from Cl0024+1654, but well-aligned with the line of sight; however, in the centre there are no galaxies which would correspond to the $z=$ 0.38 peak visible in the external distribution (Fig. 3). If the foreground component $\mathrm{B}$ were an independent loose system, it would show a deficiency of galaxies right in front of the centre of $\mathrm{Cl} 0024+1654$, which seems unlikely in the absence of interaction between the systems. This leads us to suspect that the two components are in fact physically connected and that the blue tail of the skewed central distribution originates from the same physical system as the foreground component B.

Figure 2 shows the theoretical escape velocities for masses of 1,3 and $5 \times 10^{14} M_{\odot}$ enclosed in a sphere of radius $R$. Although these lines are merely indicative due to the assumption of spherical symmetry and the unknown tangential velocity components, it is safe to say that the galaxies in component B outside, say, $600 \mathrm{~h}^{-1} \mathrm{kpc}$ are not bound to the main cluster component, unlike the negative tail of the skewed central galaxy distribution.

The galaxy density map of the main component A (Fig. 4) shows an extension of the galaxy distribution towards the northwest to a distance of $100^{\prime \prime}-150^{\prime \prime}$. This extension is visible in images of $\mathrm{Cl} 0024+1654$ and we show here that the galaxies contained in it lie at the redshift of the main cluster. Cl0024+1654 might therefore well 


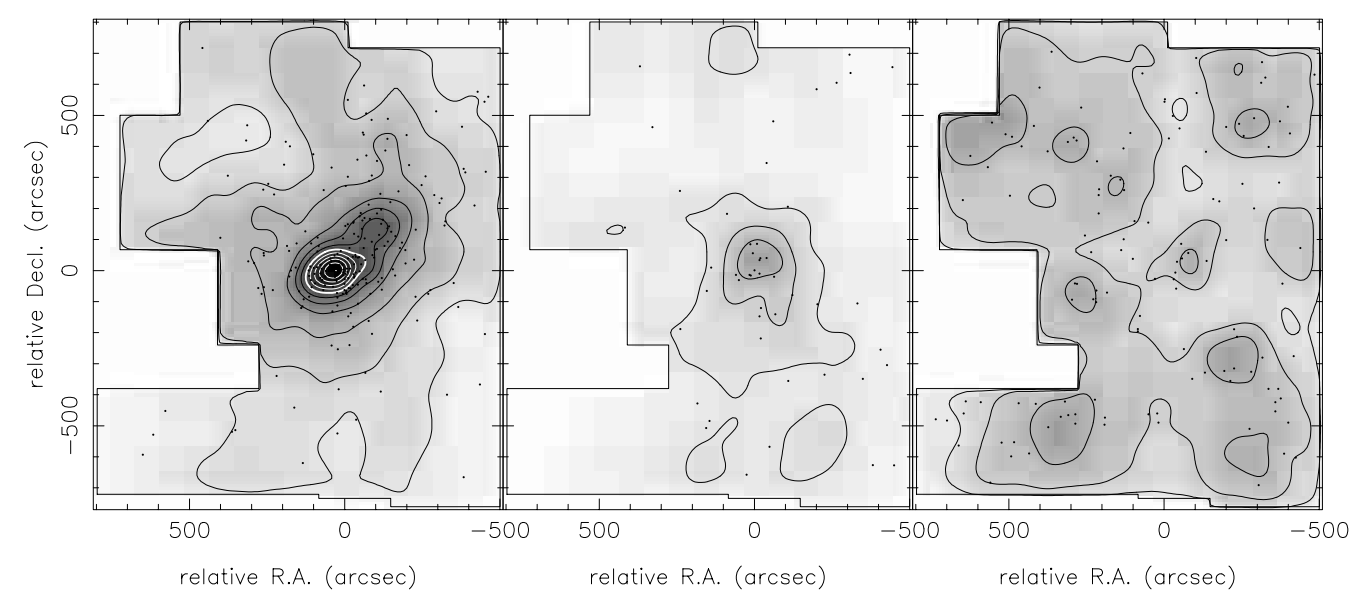

Fig. 4. Galaxy number density maps for (a) component A $(0.388<z<0.402,237$ galaxies), (b) component B $(0.37<z<0.388$, 46 galaxies) and (c) the "field" $(0.1<z<0.35,141$ galaxies), estimated with the generalized nearest neighbour method (Silverman 1986) with 10 neighbours for map (a) and 5 neighbours for maps (b) and (c). The maps are divided by the completeness map (see Paper I) and smoothed with a Gaussian with $\sigma=30^{\prime \prime}$. The grey scales are the same for all three maps. The lowest density contour lines are at $2 \%$ and $5 \%$, normalized to the maximum density in the cluster component A (left panel). The remaining contours are spaced in steps of $10 \%$. For densities below $50 \%$ the contours are drawn in black, for higher densities in white. The $50 \%$ contour is marked by a bold white line.

be comprised of three distinct subsystems: the main cluster, component A; a foreground cluster or group, component $\mathrm{B}$, which is interacting with the main cluster; and a third group or small cluster, which is falling onto the main cluster in the plane of the sky.

\subsection{Distribution of spectral types}

In Tables 1 and 2 we classify the galaxies into spectral types, defined as in Balogh et al. (1999), using our measured values for the equivalent widths of [O II], $\mathrm{H} \delta$ and the $4000 \AA$ break (see Paper I for details on how these values are defined and measured).

We consider four samples of galaxies according to redshift and projected spatial position; the sample boundaries for samples "Centre", "A" and "B" are marked by the dotted lines in Fig. 2. The boundary between "Centre" and " $\mathrm{B}$ " is to some extent arbitrary; replacing the slanted line by a simple velocity cut at $v=-1500 \mathrm{~km} \mathrm{~s}^{-1}$ does not significantly change the numbers in Tables 1 and 2, however. "Field" includes all the galaxies in the sample with redshifts $0<z<0.55$ without the cluster galaxies, $0.372<z<0.402$ (the upper limit here is the same as for the field sample in Balogh et al. 1999).

Both the classifications by $[\mathrm{O} \mathrm{II}]$ vs. $\mathrm{H} \delta$ and by $\mathrm{H} \delta$ vs. the $4000 \AA$ break show an excess of blue starforming galaxies (star-forming/short starburst, SF/SSB, and emission-line galaxies, $\mathrm{A}+\mathrm{em}$ ) in component "B" as compared to the cluster centre and possibly even over the field. The distribution of spectral types in component "A" is between a typical cluster and field populations. This seems to provide support for the hypothesis that component $\mathrm{B}$ is a loose galaxy overdensity with spectral characteristics typical for field galaxies. Alternatively it is possible that the star-formation activity in the galaxies

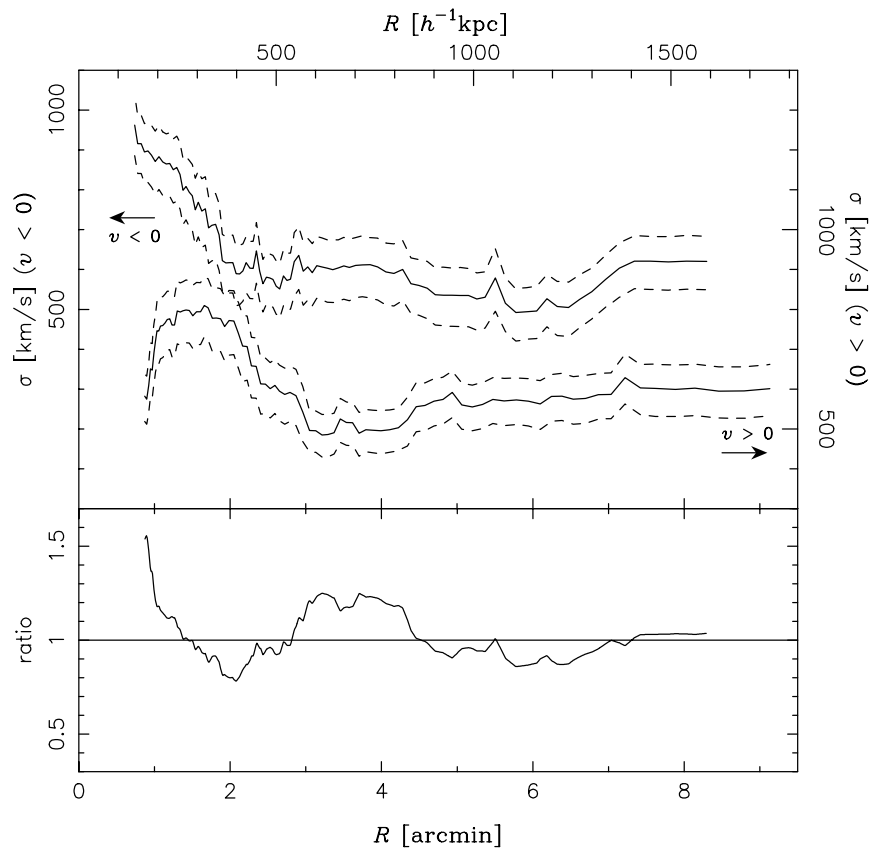

Fig. 5. Velocity dispersion profiles for galaxies with negative and positive velocity with respect to the mean redshift of cluster component $\mathrm{A}$, which was held fixed at $z_{\mathrm{A}}=0.3946$. The profiles were computed using sliding averages in bins containing 30 galaxies. The error bands give $1 \sigma$ errors determined from bootstrap resampling (10000 realisations). The curve for positive velocities was shifted by $300 \mathrm{~km} \mathrm{~s}^{-1}$ for clarity, with the corresponding velocity values marked on the right-hand axis. The bottom panel shows the ratio of the dispersions for galaxies with negative and positive velocities.

belonging to component $\mathrm{B}$ has recently been (re)activated and enhanced through interaction with a denser environment. 
Table 1. Distribution of spectral types: classification by [O II] vs. H $\delta$ (the definition of the spectral types is taken from Balogh et al. 1999 and given in Col. 2). The fraction of galaxies of a given spectral type is listed as the percentage of the total number of galaxies in the subsample (the total number is given in parentheses). See text and Fig. 2 for the definitions of the samples. The numbers are the percentages $p$ of galaxies belonging to each class in each sample. The errors are the standard deviations $\sqrt{N p(1-p)}$ of the normal distribution asymptotic to the binomial distribution with sample size $N$ and probability $p$ for large $N$.

\begin{tabular}{|c|c|c|c|c|c|c|c|}
\hline \multirow[b]{2}{*}{ Spectral Type } & \multirow[b]{2}{*}{ Definition } & \multicolumn{4}{|c|}{ This work } & \multicolumn{2}{|c|}{ Balogh et al. (1999) } \\
\hline & & Centre (183) & A (64) & B $(35)$ & Field (275) & Cluster & Field \\
\hline $\mathrm{K}+\mathrm{A}^{\mathrm{a}}$ & $\mathrm{H} \delta<-5 \AA,[\mathrm{O} \mathrm{II}]<5 \AA$ & $2 \pm 1$ & $3 \pm 2$ & 0 & $4 \pm 1$ & 4 & 2 \\
\hline $\mathrm{A}+\mathrm{em}^{\mathrm{b}}$ & $\mathrm{H} \delta<-5 \AA,[\mathrm{O} \mathrm{II}]>5 \AA$ & $3 \pm 1$ & $6 \pm 3$ & $11 \pm 5$ & $3 \pm 1$ & 4 & 10 \\
\hline $\mathrm{SF} / \mathrm{SSB}^{\mathrm{c}}$ & $\mathrm{H} \delta>-5 \AA,[\mathrm{O} \mathrm{II}]>5 \AA$ & $23 \pm 3$ & $42 \pm 6$ & $57 \pm 8$ & $53 \pm 3$ & 24 & 47 \\
\hline passive $^{\mathrm{d}}$ & $\mathrm{H} \delta>-5 \AA,[\mathrm{O} \mathrm{II}]<5 \AA$ & $72 \pm 3$ & $48 \pm 6$ & $31 \pm 7$ & $40 \pm 3$ & 67 & 41 \\
\hline
\end{tabular}

${ }^{\mathrm{a}}$ Spectrum dominated by K and A stars; ${ }^{\mathrm{b}}$ spectrum dominated by A stars, with emission lines; ${ }^{\mathrm{c}}$ star-forming/short starburst;

d red, passively evolving galaxies.

Table 2. Distribution of spectral types: classification by $\mathrm{H} \delta$ and the $4000 \AA$ break. The structure of the table is the same as for Table 1.

\begin{tabular}{|c|c|c|c|c|c|c|c|}
\hline \multirow[b]{2}{*}{ Spectral Type } & \multirow[b]{2}{*}{ Definition } & \multicolumn{4}{|c|}{ This work } & \multicolumn{2}{|c|}{ Balogh et al. (1999) } \\
\hline & & Centre (148) & A $(60)$ & B (33) & Field (225) & Cluster & Field \\
\hline $\mathrm{bHDS}^{\mathrm{a}}$ & $\mathrm{H} \delta<-5 \AA$, br $<1.5$ & $3 \pm 1$ & $3 \pm 2$ & $9 \pm 5$ & $7 \pm 1$ & 5 & 7 \\
\hline $\mathrm{rHDS}^{\mathrm{b}}$ & $\mathrm{H} \delta<-3 \AA, \mathrm{br}>1.5$ & $8 \pm 2$ & $17 \pm 5$ & $9 \pm 5$ & $5 \pm 1$ & 10 & 11 \\
\hline $\mathrm{SF} / \mathrm{SSB}^{\mathrm{c}}$ & $\mathrm{H} \delta>-5 \AA$, br $<1.5$ & $34 \pm 3$ & $50 \pm 6$ & $67 \pm 8$ & $56 \pm 3$ & 12 & 33 \\
\hline passive $^{\mathrm{d}}$ & $\mathrm{H} \delta>-3 \AA, \mathrm{br}>1.5$ & $55 \pm 4$ & $30 \pm 6$ & $15 \pm 6$ & $32 \pm 3$ & 74 & 45 \\
\hline
\end{tabular}

${ }^{\mathrm{a}}$ Blue $\mathrm{H} \delta$ strong; ${ }^{\mathrm{b}}$ red $\mathrm{H} \delta$ strong; ${ }^{\mathrm{c}}$ star-forming/short starburst; ${ }^{\mathrm{d}}$ red passively evolving galaxies.

We include in Tables 1 and 2 corresponding numbers from Balogh et al. (1999). These numbers are not strictly comparable, because the selection criteria used for the creation of our catalogue are quite different from those used by Balogh et al. (1999) ${ }^{2}$; this explains the discrepancies in particular in the $\mathrm{H} \delta$ vs. the $4000 \AA$ break classification, although the general trends are the same. The agreement in the $[\mathrm{O} \mathrm{II}]$ vs. $\mathrm{H} \delta$ classification on the other hand is very good, even quantitatively.

\section{A high speed collision?}

In general, a redshift difference between well-separated clusters at cosmological distances is a combination of a cosmological redshift difference due to the proper distance between the clusters along the line of sight and a Doppler shift due to the relative velocities of the two clusters. In general, these two effects cannot be disentangled uniquely, and there might be several ways to reproduce a given redshift distribution. In the following we develop a scenario that can reproduce the observed redshift distribution in Cl0024+1654, in particular the skewed central distribution, the presence of two well-separated modes away from the projected cluster centre extending to at least three times the virial radius.

\footnotetext{
${ }^{2}$ Balogh et al. (1999) use data from the CNOC1 cluster redshift survey, which has limiting Gunn $r$ magnitudes between 20.5 and 22.0, depending on the redshift of the observed cluster (Yee et al. 1996).
}

Assume that a group or cluster has undergone a near radial collision with $\mathrm{Cl} 0024+1654$ and we are now viewing the merging system along the direction of the impact. In fact, several characteristic parameters of a collision scenario are fairly well constrained by the observed redshift distribution. The relative velocity of the two subclusters is given by the redshift difference of components A and $\mathrm{B}$ and is thus of order $3000 \mathrm{~km} \mathrm{~s}^{-1}$. Those parts of the smaller cluster B that pass through the centre of A are more strongly decelerated in the direction of the collision than its outer parts due to stronger dynamical friction and they therefore show a smaller redshift difference. A tidal gravitational shock during the crossing scatters the outer galaxies of both clusters to large projected distance, thus increasing the extent of the cluster halos beyond their nominal virial radius. If the transverse velocity imparted on a galaxy that is now found at a radial distance of $3 h^{-1} \mathrm{Mpc}$ is $1000 \mathrm{~km} \mathrm{~s}^{-1}$, then the time since core crossing is about 3 Gyrs, and the separation between the cores of the two clusters is roughly $5 \mathrm{Mpc}$.

The main unknown in this scenario is the mass ratio between the subclusters. There are two possible versions of this collision:

(i) A small group has passed through the core of Cl0024+1654 and has been completely disrupted and scattered by the cluster's tidal force. In this case the main body of Cl0024+1654 is unperturbed and we do not observe a concentration of foreground galaxies 

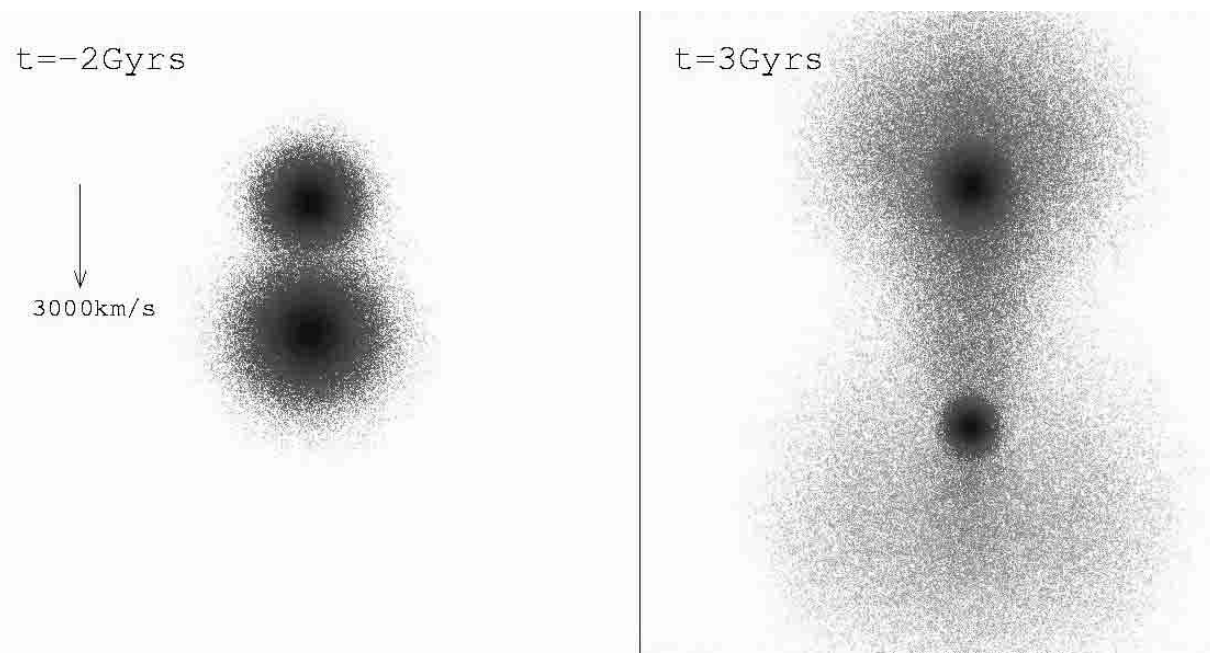

Fig. 6. The initial and final particle configurations used to simulate the two colliding clusters. The grey scale indicates the local density of dark matter within the box of length $12 \mathrm{Mpc}$.

belonging to the original group because the system has been completely unbound and is scattered to large distances perpendicular to the main cluster;

(ii) A massive cluster of approximately $50 \%$ of the mass of Cl0024+1654 collided and passed through the core of the main cluster roughly 3 Gyr ago. This collision is insufficient to completely disrupt the impacting cluster but its outer galaxies are scattered to large projected distances. The remaining bound core of the colliding cluster undergoes sufficient dynamical friction to turn its orbit around and in redshift space it appears to lie at a similar distance to $\mathrm{Cl} 0024+1654$.

Both of these scenarios can reproduce the main features of the observed redshift distribution. However, the massive collision scenario is more interesting because of the possibility of resolving both the mass discrepancy problem and the conflict between the central density structure

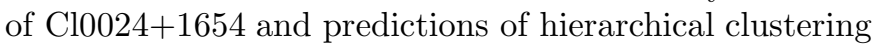
models.

The mass reconstructions of $\mathrm{Cl0024+1654}$ (Tyson et al. 1998; Broadhurst et al. 2000) using the positions of multiple images of a gravitationally lensed background galaxy show a projected mass of $1.3 \times 10^{14} h^{-1} M_{\odot}$ within $106 h^{-1} \mathrm{kpc}$ and a central surface mass density of $7900 h M_{\odot} \mathrm{pc}^{-2}$. For a singular isothermal halo extending to $r_{200} / \mathrm{kpc} \approx \sqrt{2} \sigma_{1 \mathrm{~d}} /\left(\mathrm{km} \mathrm{s}^{-1}\right)$ this implies a characteristic velocity dispersion larger than $1500 \mathrm{~km} \mathrm{~s}^{-1}$ in order to explain the projected mass.

As Shapiro \& Iliev (2000) point out, the situation is worse in the context of hierarchical clustering models. Dark matter halos in cold dark matter (CDM) type models are shallower than isothermal in their centres. In order to reproduce the observed projected mass a CDM halo with velocity dispersion of $2200 \mathrm{~km} \mathrm{~s}^{-1}$ is required. This is inconsistent with the observations presented in this paper.

A second problem for hierarchical models is that Tyson et al. (1998) infer a very shallow central density profile for this cluster, much flatter than the cuspy density profiles found for clusters in CDM type models (Ghigna et al. 2000). The central core of $\mathrm{Cl} 0024+1654$ has frequently been used to constrain the nature of dark matter and to argue for alternative candidates to CDM (Spergel \& Steinhardt 2000; Hogan \& Dalcanton 2000; Moore et al. 2000).

A high speed encounter between two similar mass clusters can explain all of the observations presented in Sect. 2 and reconcile the discrepancy between mass estimates derived for Cl0024+1654. We now explore this scenario using high resolution numerical simulations of colliding dark matter halos to study the evolution of the mass distribution. We construct two equilibrium $\mathrm{CDM}$ halos with virial masses $9.5 \times 10^{14} M_{\odot}$ and $5.0 \times 10^{14} M_{\odot}$ with peak circular velocities $v_{\text {peak }}=1600$ and $1300 \mathrm{~km} \mathrm{~s}^{-1}$ and concentrations $c=5$ and $c=7$ respectively. Their initial separation is $3 \mathrm{Mpc}$ and relative velocity is $-3000 \mathrm{~km} \mathrm{~s}^{-1}$. The particle mass is set to $5 \times 10^{9} M_{\odot}$ and we use an equivalent Plummer force softening of $5 \mathrm{kpc}$.

After the collision we find that the outer regions of the smaller cluster have become unbound and are streaming radially away from the impact location. The impulse velocity perpendicular to the encounter is of the order $1000 \mathrm{~km} \mathrm{~s}^{-1}$. Snapshots of the initial and final times are shown in Fig. 6. The peak circular velocities of the bound components have fallen to 1430 and $1140 \mathrm{~km} \mathrm{~s}^{-1}$ whilst the central $1 \mathrm{D}$ velocity dispersions have reduced to 930 and $710 \mathrm{~km} \mathrm{~s}^{-1}$ from initial values of 1033 and $883 \mathrm{~km} \mathrm{~s}^{-1}$ respectively. At the final time we find a total mass within a cylinder of radius $106 \mathrm{kpc}$ of $8.0 \times 10^{13} M_{\odot}$.

The redshift distribution of 10000 randomly selected particles at the final time is shown in the right hand panel in Fig. 7. This is not a perfect match to the observational data but the main features are present: we see a foreground component of "galaxies" that span large projected distances from the central region of Cl0024+1654. This component is separated by $\approx 3000 \mathrm{~km} \mathrm{~s}^{-1}$ in redshift space from the main component. The smaller cluster bound core 

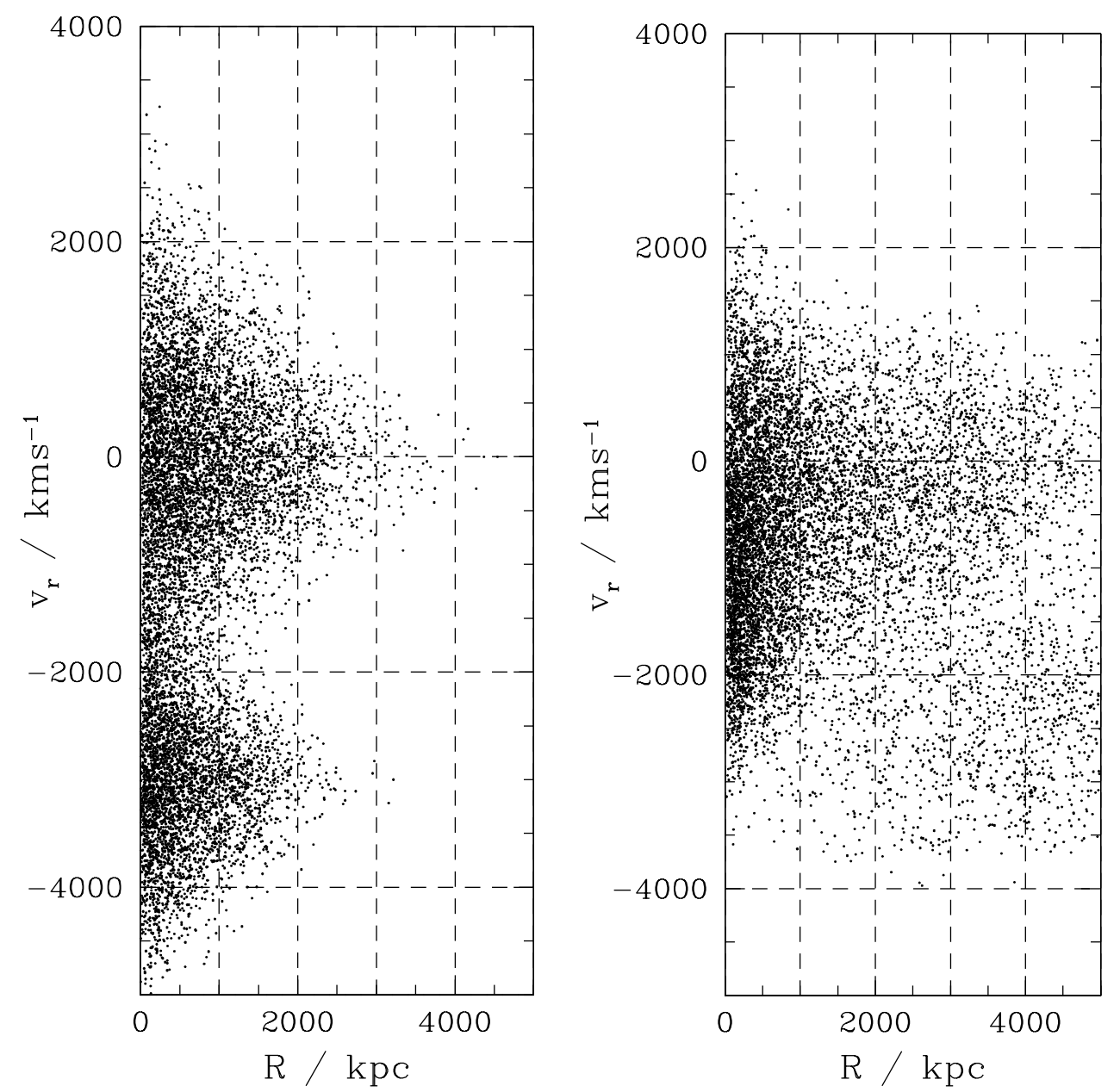

Fig. 7. The "observed" redshift distribution of particles in the collision between the simulated clusters. The left panel shows the initial conditions whilst the right panel shows the data 3 Gyrs after the collision. This is to be compared with Fig. 2.

is moving away from the main cluster at $\approx 1000 \mathrm{~km} \mathrm{~s}^{-1}-$ the reduction in speed from the initial velocity is due to dynamical friction. A better agreement would result if the impacting cluster was slightly more massive in which case it would suffer more friction and would be seen nearly at rest compared to the main component. Also note that we are plotting "dark matter particles" not "galaxies". Of course the initial configuration shown in the left panel in Fig. 7 also displays a bimodal distribution of redshifts. However, this configuration is more symmetric than either the final simulated configuration or the observed redshift distribution; also, the radial extent, in particular of the foreground (in redshift space) component, is much smaller than observed.

The energy from the impulsive tidal shock has been transferred into kinetic energy of the particles, heating and expanding the cold central cores of the clusters. This leads to a flattening of the physical and projected density profiles. In Fig. 8 we show the projected surface mass density profile before and after the encounter. The initial profiles are cuspy, CDM type density profiles whereas the final profiles have nearly constant density cores in good agreement with that inferred from the mass reconstruction (Tyson et al. 1998, their Fig. 4). The central surface mass density is $4000 M_{\odot} \mathrm{pc}^{-2}$ which is very close to the value obtained by Tyson et al. for $H_{0}=50 \mathrm{~km} \mathrm{~s}^{-1} \mathrm{Mpc}^{-1}$. The total projected mass within the central $106 \mathrm{kpc}$ is about $30 \%$ lower than obtained by Broadhurst et al. (2000) which could be reconciled by using a more massive encounter and/or properly including the baryonic matter.

\section{Comparison to other observations}

The galaxy distribution of $\mathrm{Cl} 0024+1654$ in redshift space and on the sky, as described in Sect. 2, provides strong hints that this is not a simple relaxed system. How do the skewed central velocity dispersion and the presence of a foreground component in redshift space affect the interpretation of other observations and in particular mass estimates of this system?

\subsection{Galaxy dynamics}

Dynamical mass estimates rely on the assumption that the galaxies are in virial equilibrium in the cluster's gravitational potential well and that their velocities are purely random. 


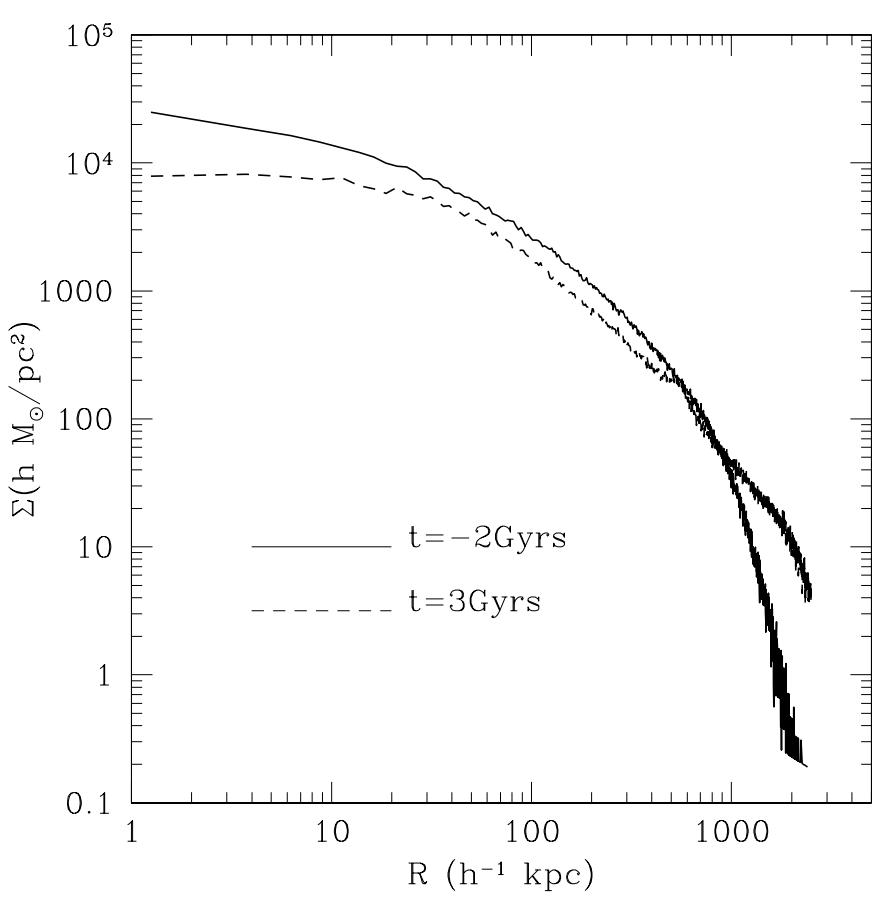

Fig. 8. The surface mass density profiles projected along the merger axis before (solid curve) and after the collision (dashed curve). The central surface mass density after the collision is very close to that measured by Tyson et al. (1998).

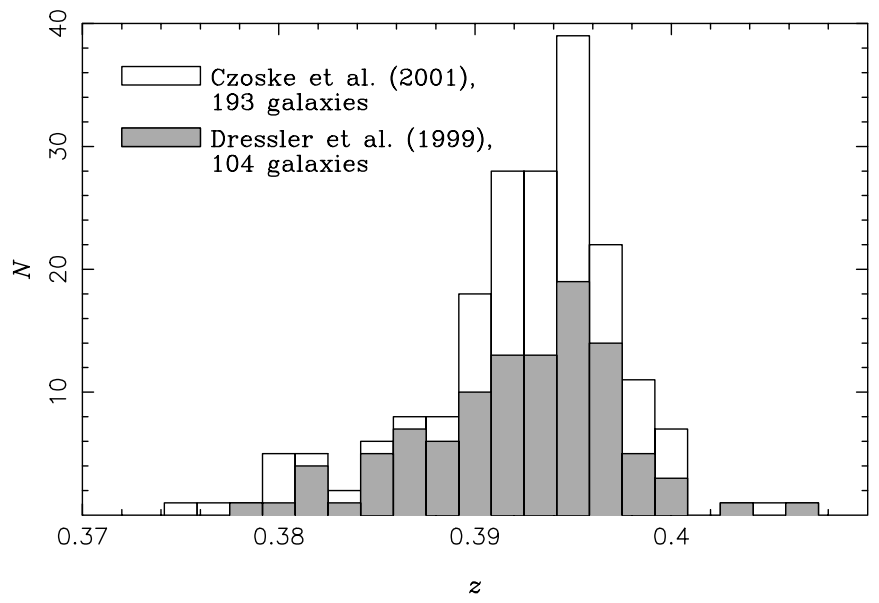

Fig. 9. Comparison of redshift histograms of the data of D99 (corrected for the misidentifications discussed in Paper I) and Paper I (these include the data of D99), constrained to the area covered by D99, clustercentric distance $<5^{\prime}$. Bin widths and centres are the same as in the inset of Fig. 2 of D99.

The large velocity dispersion of $\sigma \approx 1200 \mathrm{~km} \mathrm{~s}^{-1}$ found by Dressler \& Gunn (1992) and Dressler et al. (1999) (hereafter D99) was based on galaxies within $5^{\prime}$ from the projected cluster centre. As shown in Fig. 9, there is no direct evidence for the bimodality of $\mathrm{Cl} 0024+1654$ when attention is restricted to the central regions of the cluster. This is still true with the larger number of galaxy spectra used here: Whereas our catalogue contains about $85 \%$ more redshifts in this area than D99, the general shape of the histogram is the same. Both histograms are strongly skewed towards low redshifts, thus indicating the presence of substructure (Ashman et al. 1994) or a bulk velocity component. Note that the bimodality of the redshift distribution only becomes apparent when galaxies at larger projected distances from the cluster centre are included. In this case it is therefore not the number of galaxy redshifts but their distribution over a wide field that provides the clues to the complexity of this system.

The formal velocity dispersion for the 193 galaxies within $5^{\prime}$ (Fig. 9) is $\sigma_{\text {cent }}=1050 \mathrm{~km} \mathrm{~s}^{-1}$. If there is indeed a bulk velocity component present in the central velocity distribution, then mass estimates based on the formal central velocity dispersion will overestimate the true cluster mass.

A better dynamical mass estimate might be obtained from the velocity dispersion at larger radii, where the two components A and B are clearly separated. At projected distances $>3^{\prime}$ the velocity distribution of component $\mathrm{A}$ is regular with a dispersion of $\sim 600 \mathrm{~km} \mathrm{~s}^{-1}$. If we interpreted this value as the velocity dispersion of a relaxed cluster, it would have only about a quarter of the mass previously estimated for Cl0024+1654 (Schneider et al. 1986), below mass estimates from gravitational lensing (which are roughly consistent with a high velocity dispersion) and even below mass estimates from X-ray observations. In the context of the collision scenario presented in Sect. 3 on the other hand, the galaxies at large projected distance from the cluster centre are also affected by the collision and cannot be used to derive a mass estimate based on the assumption of dynamical equilibrium.

\subsection{X-ray observations}

The X-ray observations from Rosat/HRI and ASCA (Soucail et al. 2000; Böhringer et al. 2000) by themselves show no indication of anything other than a fairly small cluster of galaxies. Given the measured values for $\mathrm{Cl0024+1654,} T_{\mathrm{X}}=5.7_{-2.1}^{+4.9} \mathrm{keV}$ and $L_{\mathrm{X}}=6.8 \times$ $10^{43} h^{-2} \mathrm{erg} \mathrm{s}^{-1}$ (Soucail et al. 2000), the gas does not seem too far away from the $L_{\mathrm{X}}-T_{\mathrm{X}}$ relation of Markevitch (1998); it is slightly too hot for its luminosity. The morphology is regular and the surface brightness profile well fitted by a beta profile with a surprisingly small core radius of $33 h^{-1} \mathrm{kpc}$. Whereas no significant substructure is required to model the X-ray observations of $\mathrm{Cl} 0024+1654$, they do not provide evidence against the collision scenario either.

The X-ray emission is difficult to predict in the context of two clusters projected along the line-of-sight several Gyr after a head-on collision. During the collision, hydrostatic equilibrium in the gas component of the cluster(s) breaks down and the X-ray luminosity and emissionweighted temperature fluctuate considerably (by up to a factor of 10 for $L_{\mathrm{X}}$ ) and rapidly as a consequence of the formation of shock waves and repeated expansion and compression of the core gas (Takizawa 1999; Ricker \& Sarazin 2001; Ritchie \& Thomas 2001). After several Gyr 
however, shocks have dissipated and the gas settles down to an equilibrium configuration. The cited hydrodynamic simulations of cluster mergers consider collision speeds of about $1000 \mathrm{~km} \mathrm{~s}^{-1}$; in this case the dark matter cores separate to distances of only a couple of Mpc before turning around and eventually merging into one clump. In a high-speed collision, however, the collisionless dark matter cores of the two clusters separate to large distance before turning around. The gas, due to its collisional nature should experience stronger interaction during the first crossing and thus behave quite differently from the dark matter/galaxies and possibly also from what low-speed collision simulations predict. (Ricker \& Sarazin 2001) observe a slight segregation between the gas and the dark matter cores in their simulations. This effect should be more pronounced in a high-speed collision.

If we accept the collision scenario then a mass estimate from the X-ray observations is not possible. Detailed inclusion of a hydrodynamic treatment of the gas component in simulations of high-speed collisions is necessary to understand the behaviour of the X-ray emission in this case. High resolution X-ray imaging with CHANDRA has recently been obtained (P. I. Hattori) but has not been published yet.

\subsection{Gravitational lensing}

Gravitational lensing by clusters of galaxies does not rely on the matter in the clusters being in dynamical equilibrium. However, gravitational lensing measures the weighted integral of all the mass between the observer and the source, and the interpretation of the measured mass value thus depends on the detailed distribution of mass along the line-of-sight. The skew in the central redshift distribution in Cl0024+1654 is an indication of bulk motion, possibly due to a high-speed collision between two fairly massive clusters of a mass ratio of roughly $2: 1$, or by the passage of a small group through the core of Cl0024+1654. In the latter case the mass determined by strong lensing models would indeed be indicative of the mass of the main cluster; however, in this case one would expect the X-ray emitting gas to be largely unperturbed by the collision, and one would be left with the discrepancy between the mass estimates derived from lensing and X-rays. In the former case, the lensing mass would be the sum of the two cluster cores and therefore too large if interpreted as representing the mass of a single cluster.

The projected mass profile for the post-merger system seen in our simulations shows two characteristics which are testable by gravitational lensing analyses of $\mathrm{Cl} 10024+1654$ : At small scales of several 10's kpc, the profile shows a flat core which is consistent with strong lensing mass models (Tyson et al. 1998), as shown in Sect. 3. On large scales the profile is significantly flatter than the initial CDM profile, falling only as $r^{-2.5}$ out to $\sim 3 \mathrm{Mpc}$. Previous weak lensing analyses on $\mathrm{Cl} 10024+1654$ have not attempted a detailed reconstruction of the mass profile. Bonnet et al. (1994) and van Waerbeke et al. (1997) derived shear maps from ground-based data on the north-east quadrant of the cluster, Smail et al. (1997) were restricted to the small field of view of HST/WFPC2. The shear pattern found by Bonnet et al. (1994) is compatible with an isothermal sphere profile, $\rho \propto r^{-2}$ out to $3 h^{-1} \mathrm{Mpc}$.

A weak lensing analysis of newly obtained CFHT/CFH12k images in BVRI is currently underway (Czoske et al. 2002, in preparation). Also, a sparsely sampled HST/WFPC2 mosaic consisting of 38 pointings out to a distance of $2.5 h^{-1} \mathrm{Mpc}$ from the cluster centre has recently been obtained; these data will also be used for a weak lensing analysis (Treu et al. 2001). These data will allow to probe the cluster's projected radial mass profile accurately to the edge of the survey field.

\section{Discussion and conclusions}

In the present paper we have analyzed the galaxy distribution in the cluster of galaxies Cl0024+1654, based on $\sim 300$ galaxy redshifts and projected positions. The cluster, which was previously regarded as a prototype of a massive relaxed cluster at intermediate redshift, turns out to have a fairly complicated structure, showing a strongly skewed redshift distribution in its central parts and two well-separated components at larger projected distances out to $\sim 3 h^{-1} \mathrm{Mpc}$.

We argue that the blue tail of the central velocity distribution and the foreground component originate from the same physical system and interpret the peculiar redshift-space distribution as the result of a high-speed head-on collision of two clusters of galaxies, the merger axis being very nearly parallel to the line-of-sight. Using a numerical simulation we show that it is possible to explain the observed redshift distribution with a high-speed collision of two rather massive clusters of galaxies with a mass ratio of about $2: 1$.

Apart from reproducing the spatial/redshift distribution of the cluster galaxies, this scenario also produces a projected mass distribution which is very close to that derived by Tyson et al. (1998) from the quintuple arc system observed in $\mathrm{Cl} 0024+1654$. Note that the scenario was not designed to reproduce this mass profile. Since it is thus possible to produce a mass distribution with a flat core from the merger of two CDM halos this eliminates one of the main arguments against simple non-interacting cold dark matter as the dynamically dominant component in clusters of galaxies (Spergel \& Steinhardt 2000).

The distribution of spectral types in the foreground component B is more akin to the general field population than to a cluster population. In the context of our collision scenario, these would correspond to the outer regions of the smaller cluster which have become unbound during the impact. Even initially, these galaxies would probably not correspond to a fully transformed cluster galaxy population. In addition, the impact might have triggered additional star formation in these galaxies. Remembering

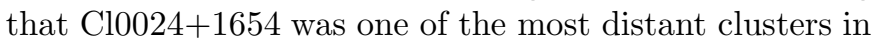


which the Butcher-Oemler effect was detected (Butcher \& Oemler 1984; Dressler et al. 1985), this may add a new view on this effect: The fact that $\sim 40 \%$ of the bright galaxies in the cluster are emission line galaxies ([O II $]>5 \AA$ ) has a natural interpretation in the collision scenario. The spectral distribution of the galaxies in component A (and centre) are also perturbed by the collision with a possible excess of rHDS and SF/SBB (Tables 1 and 2); rHDS galaxies may represent the result of a starburst induced during the early stages of the interaction.

The relative velocity of the clusters of $\sim 3000 \mathrm{~km} \mathrm{~s}^{-1}$ implied by the redshift distribution in $\mathrm{Cl} 10024+1654$ is very high. Observations and simulations find mean peculiar velocities for clusters of order $500 \mathrm{~km} \mathrm{~s}^{-1}$ (Bahcall \& Oh 1996; Giovanelli et al. 1998; Gibbons et al. 2001; Colberg et al. 2000). Still, colliding clusters can reach relative velocities of about $3000 \mathrm{~km} \mathrm{~s}^{-1}$ at separations of about $1 \mathrm{Mpc}$ (Sarazin 2001). Markevitch et al. (2001) have recently found a bow shock in the galaxy cluster 1E0657-56 which implies a relative speed of 3000 to $4000 \mathrm{~km} \mathrm{~s}^{-1}$ for the collision. Cl0024+1654 is still an extraordinary but not an impossible system.

Acknowledgements. OC thanks the European Commission for generous support under grant number ER-BFM-BI-CT97-2471 and the Institute for Astronomy in Honolulu, in particular Harald Ebeling, for kind hospitality. JPK thanks CNRS for support. We also acknowledge support from the UKFrench ALLIANCE collaboration programme 0161XM and support from the TMR Network "Gravitational Lensing: New Constraints on Cosmology and the Distribution of Dark Matter" of the European Commission under contract No. ERBFM-RX-CT97-0172. We thank the referee, Richard Ellis, for his comments which helped improve the paper.

\section{References}

Ashman, K. A., Bird, C. M., \& Zepf, S. E. 1994, AJ, 108, 2348 Bahcall, N. A., \& Oh, S. P. 1996, ApJ, 462, L49

Balogh, M. L., Morris, S. L., Yee, H. K. C., Carlberg, R. G., \& Ellingson, E. 1999, ApJ, 527, 54

Beers, T. C., Flynn, K., \& Gebhardt, K. 1990, AJ, 100, 32

Böhringer, H., Soucail, G., Mellier, Y., Ikebe, Y., \& Schuecker, P. 2000, A\&A, 353, 124

Bonnet, H., Mellier, Y., \& Fort, B. 1994, ApJ, 427, L83

Broadhurst, T., Huang, X., Frye, B., \& Ellis, R. S. 2000, ApJ, 534, L15

Butcher, H., \& Oemler Jr., A. 1984, ApJ, 285, 426

Colberg, J. M., White, S. D. M., MacFarland, T. J., et al. 2000, MNRAS, 313, 229

Czoske, O., Kneib, J.-P., Soucail, G., et al. 2001, A\&A, 372, 391

Dressler, A., \& Gunn, J. E. 1992, ApJS, 78, 1

Dressler, A., Gunn, J. E., \& Schneider, D. P. 1985, ApJ, 294, 70
Dressler, A., Smail, I., Poggianti, B. M., et al. 1999, ApJS, 122, 51

Ghigna, S., Moore, B., Governato, F., et al. 2000, ApJ, 544, 616

Gibbons, R. A., Fruchter, A. S., \& Bothun, G. D. 2001, AJ, 121,649

Giovanelli, R., Haynes, M. P., Salzer, J. J., et al. 1998, AJ, 116, 2632

Girardi, M., \& Mezzetti, M. 2001, ApJ, 548, 79

Hogan, C. J., \& Dalcanton, J. J. 2000, Phys. Rev. D, 62, 063511

Kassiola, A., Kovner, I., \& Fort, B. 1992, ApJ, 400, 41

Markevitch, M. 1998, ApJ, 504, 27

Markevitch, M., Gonzalez, A. H., David, L., et al. 2001 [astro-ph/0110468]

Markevitch, M., Ponman, T., Nulsen, P. E. J., et al. 2000, ApJ, 541,542

Markevitch, M., \& Vikhlinin, A. 2001 [astro-ph/0105093]

Mazzotta, P., Markevitch, M., Vikhlinin, A., et al. 2001, ApJ, 555,205

Miralda-Escudé, J., \& Babul, A. 1995, ApJ, 449, 18

Moore, B., Gelato, S., Jenkins, A., Pearce, F. R., \& Quilis, V. 2000, ApJ, 535, L21

Moore, B., Governato, F., Quinn, T., Stadel, J., \& Lake, G. 1998, ApJ, 499, L5

Navarro, J. F., Frenk, C. S., \& White, S. D. M. 1997, ApJ, 490, 493

Press, W. H., Teukolsky, S. A., Vetterling, W. T., \& Flannery, B. P. 1992, Numerical Recipes in C, 2nd ed. (Cambridge University Press)

Ricker, P. M., \& Sarazin, C. L. 2001 [astro-ph/0107210]

Ritchie, B. W., \& Thomas, P. A. 2001 [astro-ph/0107374]

Sarazin, C. L. 2001, in Merging Processes in Clusters of Galaxies, ed. L. Feretti, I. M. Gioia, \& G. Giovannini (Kluwer, Dordrecht) [astro-ph/0105418]

Schneider, D. P., Dressler, A., \& Gunn, J. E. 1986, AJ, 92, 523

Shapiro, P. R., \& Iliev, I. T. 2000, ApJ, 542, L1

Shapiro, S. S., \& Wilk, M. B. 1965, Biometrika, 52, 591

Silverman, B. W. 1986, Density Estimation for Statistics and Data Analysis, Monographs on Statistics and Applied Probability (London: Chapman and Hall)

Smail, I., Dressler, A., Kneib, J.-P., et al. 1996, ApJ, 469, 508

Smail, I., Ellis, R. S., Dressler, A., et al. 1997, ApJ, 479, 70

Soucail, G., Ota, N., Böhringer, H., et al. 2000, A\&A, 355, 433

Spergel, D. N., \& Steinhardt, P. J. 2000, Phys. Rev. Lett., 84, 3760

Takizawa, M. 1999, ApJ, 520, 514

Treu, T., Ellis, R. S., Trivedi, P., et al. 2001, in SESTO2001-Tracing Cosmic Evolution with Galaxy Clusters, ASP Conf. Ser. [astro-ph/0112409].

Tyson, J. A., Kochanski, G. P., \& Dell'Antonio, I. P. 1998, ApJ, 498, L107

van Waerbeke, L., Mellier, Y., Schneider, P., Fort, B., \& Mathez, G. 1997, A\&A, 317, 303

Vikhlinin, A., Markevitch, M., \& Murray, S. S. 2001, ApJ, 551, 160

Wu, X.-P., Chiueh, T., Fang, L.-Z., \& Xue, Y.-J. 1998, MNRAS, 301, 861

Yee, H. K. C., Ellingson, E., \& Carlberg, R. G. 1996, ApJ, 102, 269 